\title{
Effect of adaptive statistical iterative reconstruction-V (ASiR-V) levels on ultra-low-dose CT radiomics quantification in pulmonary nodules
}

\author{
Kai Ye ${ }^{1}$, Min Chen ${ }^{2}$, Qiao Zhu' ${ }^{1}$, Yuliu Lu ${ }^{1}$, Huishu Yuan ${ }^{1}$ \\ ${ }^{1}$ Department of Radiology, Peking University Third Hospital, Beijing, China; ${ }^{2}$ Department of Radiology, Ghent University Hospital, Corneel \\ Heymanslaan 10,9000, Ghent, Belgium \\ Correspondence to: Huishu Yuan. Department of Radiology, Peking University Third Hospital, 49 North Garden Road, Haidian District, Beijing \\ 100191, China. Email: huishuy@bjmu.edu.cn.
}

Background: The weightings of iterative reconstruction algorithm can affect CT radiomic quantification. But, the effect of ASiR-V levels on the reproducibility of CT radiomic features between ultra-low-dose computed tomography (ULDCT) and low-dose computed tomography (LDCT) is still unknown. The purpose of study is to investigate whether adaptive statistical iterative reconstruction- $\mathrm{V}$ (ASiR-V) levels affect radiomic feature quantification using ULDCT and to assess the reproducibility of radiomic features between ULDCT and LDCT.

Methods: Sixty-three patients with pulmonary nodules underwent LDCT $(0.70 \pm 0.16 \mathrm{mSv})$ and ULDCT $(0.15 \pm 0.02 \mathrm{mSv})$. LDCT was reconstructed with ASiR-V 50\%, and ULDCT with ASiR-V 50\%, 70\%, and $90 \%$. Radiomics analysis was applied, and 107 features were extracted. The concordance correlation coefficient (CCC) was calculated to describe agreement among ULDCTs and between ULDCT and LDCT for each feature. The proportion of features with CCC $>0.9$ among ULDCTs and between ULDCT and LDCT, and the mean CCC for all features between ULDCT and LDCT were also compared.

Results: Sixty-three solid nodules (SNs) and 48 pure ground-glass nodules (pGGNs) were analyzed. There was no difference for the proportion of features in SNs among ULDCTs and between ULDCT and LDCT $(\mathrm{P}>0.05)$. The proportion of features in pGGNs were highest for $\mathrm{ULDCT}_{70 \% \text { vs. } 90 \%}(78.5 \%)$ and $\mathrm{ULDCT}_{90 \%}$ vs. $\mathrm{LDCT}_{50 \%}(50.5 \%)$. In SNs, the mean CCC for $\mathrm{ULDCT}_{90 \%}$ vs. $\mathrm{LDCT}_{50 \%}$ was $0.67 \pm 0.26$, not different with that for $\mathrm{ULDCT}_{50 \%} v$ s. $\mathrm{LDCT}_{50 \%}(0.68 \pm 0.24)$ and $\mathrm{ULDCT}_{70 \%} v s . \mathrm{LDCT}_{50 \%}(0.64 \pm 0.21)(\mathrm{P}>0.05)$. In pGGNs, the mean CCC for $\mathrm{ULDCT}_{90 \%} v s$. $\mathrm{LDCT}_{50 \%}$ was $0.79 \pm 0.19$, higher than that for $\mathrm{ULDCT}_{50 \%} v s$. $\mathrm{LDCT}_{50 \%}(0.61 \pm 0.28)$ and $\mathrm{ULDCT}_{70 \%} v s . \mathrm{LDCT}_{50 \%}(0.76 \pm 0.24)(\mathrm{P}<0.05)$.

Conclusions: ASiR-V levels significantly affected ULDCT radiomic feature quantification in pulmonary nodules, with stronger effects in pGGNs than in SNs. The reproducibility of radiomic features was highest between $\mathrm{ULDCT}_{90 \%}$ and $\mathrm{LDCT}_{50 \%}$.

Keywords: Computed X-ray tomography; radiomics; pulmonary nodule; ASiR-V; reproducibility

Submitted Jul 31, 2020. Accepted for publication Dec 22, 2020.

doi: 10.21037/qims-20-932

View this article at: http://dx.doi.org/10.21037/qims-20-932 


\section{Introduction}

In recent years, the development of computational power and image feature extraction has led to the use of highthroughput extraction of quantitative imaging features combined with different kinds of diagnostic models to assist clinical decisions, in a process called radiomics $(1,2)$. Extensive research indicates that radiomics has shown promise in pulmonary nodule characterization, histopathologic staging, prognostic analysis, and recognition of gene mutations (3-7).

At present, chest low-dose computed tomography (LDCT) is the recommended modality for screening non-small cell lung cancer (NSCLC) (8). In addition to monitoring the size of pulmonary nodules, LDCT is also widely used in radiomics analysis (9-11). Owing to developments in computed tomography, the wide applicability of iterative reconstruction (IR) algorithms has enabled the realization of ultra-low-dose CT (ULDCT), which has a lower radiation dose than LDCT. The radiation dose of ULDCT is $<0.2 \mathrm{mSv}$, which is comparable with the dose of chest radiography (12-14). As a third-generation IR, adaptive statistical iterative reconstruction-V (ASiR-V) has the ability to reduce image noise and is widely adopted in the image reconstruction of ULDCT $(15,16)$.

Recently, there have been many reports on the reliability of ULDCT for screening NSCLC, with some researchers even recommending replacing LDCT with ULDCT for NSCLC screening (17-20). Nevertheless, these studies focused on conventional image diagnosis by the naked eye and ignored the ULDCT radiomic quantification in pulmonary nodules in vivo. The reproducibility of CT radiomic features between LDCT and ULDCT remains unknown. Moreover, some studies have shown that changing the weightings of IR can affect the reproducibility of CT radiomic features (21). However, there are only a few studies on the effect of IR algorithms on CT radiomic quantification considering the high number of commercial IR algorithms that are composed of different models (22-26). The effect of changing the weightings for different IR algorithms on the reproducibility of $\mathrm{CT}$ radiomic features needs to be investigated further.

Therefore, this study aimed to evaluate the variability of quantification of radiomic features computed using ULDCT reconstructed with different ASiR-V levels and investigate the effect of ASiR-V levels on the reproducibility of CT radiomic features between ULDCT reconstructed with different ASiR-V levels and LDCT, by assessing the agreement of CT radiomic features (including first-order statistical, textural, and structural features) among ULDCTs with different ASiR-V levels as well as the agreement between ULDCT and LDCT in pulmonary nodules.

\section{Methods}

\section{Participants}

From August 2019 to January 2020, we enrolled patients who underwent LDCT for pulmonary nodule follow-up in our radiology department. The inclusion criteria were as follows: (I) age $\geq 18$ years and body mass index (BMI) $\leq$ $35 \mathrm{~kg} / \mathrm{m}^{2}$, (II) acceptable diagnostic image quality of LDCT, (III) $\leq 5$ pulmonary nodules without calcification, and (IV) solid nodules (SNs) and pure ground-glass nodules (pGGNs) with diameters of 4-15 $\mathrm{mm}$ (the diameter was calculated as the mean of the longest diameter and perpendicular diameter of a nodule) (27). The exclusion criteria were as follows: (I) LDCT with the tube voltage of $100 \mathrm{kV}$, (II) patients with diffuse consolidation and/or other diseases, making pulmonary nodules unevaluable, and (III) patients with pulmonary part-solid nodules (PSNs).

There were 85 patients in the preliminary study. Among them, twenty-two patients were excluded (three patients with poor image quality of LDCT, five patients with PSNs and fourteen patients scanned with tube voltage of $100 \mathrm{kV}$ ). Finally, our study population consisted of 63 patients [14

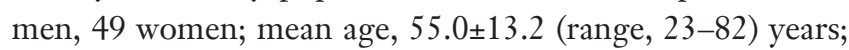
mean BMI, $22.67 \pm 2.95$ (range, $\left.17.5-31.14) \mathrm{kg} / \mathrm{m}^{2}\right]$.

This study was approved by the institutional review board of our department, and informed consent was obtained from the participants.

\section{CT acquisition and reconstruction}

All CT scans were performed on a revolution CT scanner (GE Healthcare, Milwaukee, WI, USA) and conducted with the patient in a deep inspiratory breath-hold. Patients were scanned using a conventional non-enhanced LDCT protocol, immediately followed by ULDCT. The interval between the two protocols was $<1 \mathrm{~min}$. All scans were used in helical mode, with a scan range from the costophrenic angle to the pulmonary apex.

The LDCT dataset was obtained with a collimation of $64 \times 0.625 \mathrm{~mm}$, beam pitch of $0.984: 1$, Assist tube voltage of $120 / 100 \mathrm{kV}$, Smart mA with a noise index of $14.1 \mathrm{HU}$ $(\mathrm{min} / \mathrm{max} \mathrm{mA}, 50 / 680)$, and gantry rotation time of $0.28 \mathrm{~s}$. 


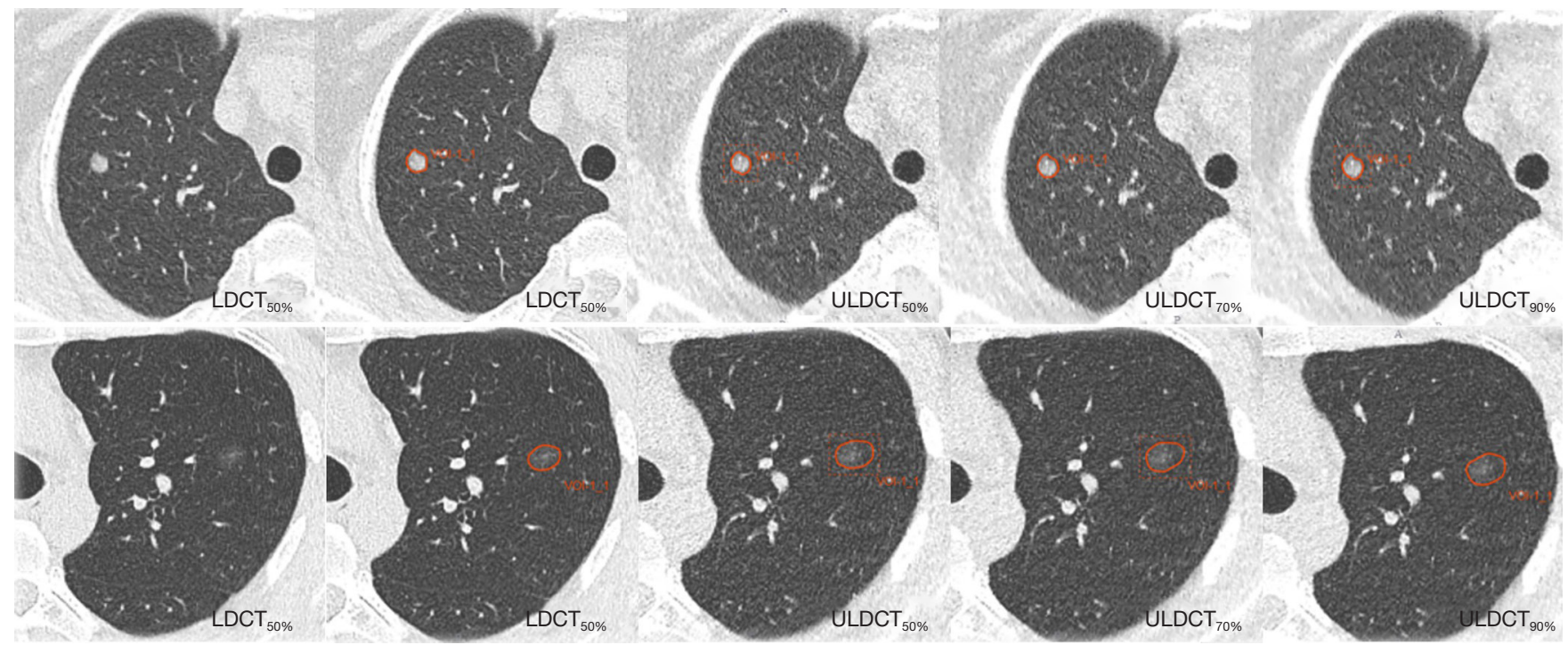

Figure 1 Process of nodule segmentation for both solid nodule and pure ground-glass nodule. ULDCT ${ }_{50 \%}$, ultra-low-dose CT (ULDCT) with ASiR-V 50\%; ULDCT $_{70 \%}$, ULDCT with ASiR-V 70\%; ULDCT $90 \%$, ULDCT with ASiR-V 90\%; LDCT $_{50 \%}$, low-dose CT (LDCT) with ASIR-V $50 \%$.

Identical parameters were used for ULDCT, except that the tube voltage was fixed at $120 \mathrm{kV}$ and the tube current was Smart $\mathrm{mA}$ with a noise index of 28 HU. LDCT images were reconstructed with ASiR-V 50\%, which was described as $\mathrm{LDCT}_{50 \%}$. ULDCT images were reconstructed with ASiR-V 50\%, 70\%, and 90\%, which were described as $\mathrm{ULDCT}_{50 \%}, \mathrm{ULDCT}_{70 \%}$, and ULDCT $90 \%$, respectively. ASiR-V levels used for the image reconstruction of ULDCT were based on the results of our previous study (published in Chinese domestic core journal). All images were obtained using a slice thickness of $1.25 \mathrm{~mm}$ with an increment of $1.25 \mathrm{~mm}$. The image matrix was $512 \times 512$ pixels.

The mean CT dose index volume (CTDIvol) and doselength product (DLP) for LDCT were $1.4 \pm 0.33 \mathrm{mGy}$ and $49.83 \pm 11.76 \mathrm{mGy} \cdot \mathrm{cm}$, respectively. The mean CTDIvol and DLP for ULDCT were $0.33 \pm 0.16$ and $11.05 \pm 1.7 \mathrm{mGy}$ $\mathrm{cm}$, respectively. The effective dose (ED) was calculated by multiplying the DLP with a conversion coefficient $\mathrm{k}$ of $0.014 \mathrm{mSv} / \mathrm{mGy} / \mathrm{cm}(28)$.

\section{Nodule segmentation and radiomic feature extraction}

All patient identifiers (including name, age, sex, and medical record number) were removed from the images. Images were presented with a fixed window center of $-600 \mathrm{HU}$ and width of 1,600 HU. Image quality of LDCT was assessed by a radiologist (Doctor A with 5 years' experience in radiology). Images with moderate or mild artefacts which do not affect diagnosis were seen as acceptable. Pulmonary nodule segmentation was performed by two radiologists (Doctor B with 6 years' experience in chest CT and Doctor C with 8 years' experience) in consensus using a semiautomatic segmentation and radiomic feature extraction tool (RadCloud version 3.0; Huiying Medical Technology Co., Ltd.). If the discrepancy of volumes of interest drawn by two doctors was more than 5\% (29), the segmentation performed by a third reader (Doctor D with 26 years' experience in chest CT) was chosen. The process of nodule segmentation for both SNs and pGGNs is shown in Figure 1.

After nodule segmentation, a total of 107 radiomic features from 3D data were extracted, including histogram, texture and structural features. They included 18 first-order statistical features (histogram), 75 texture features [graylevel co-occurrence matrix (GLCM), gray-level dependence matrix (GLDM), gray-level run length matrix (GLRLM), gray-level size zone matrix (GLSZM), neighboring graytone difference matrix (NGTDM)], and 14 structural features (Shape-3D). All of these features were directly extracted from the original images without filtering and can intuitively reflect original information of images. A detailed description of all these 107 features is shown in Table S1. 


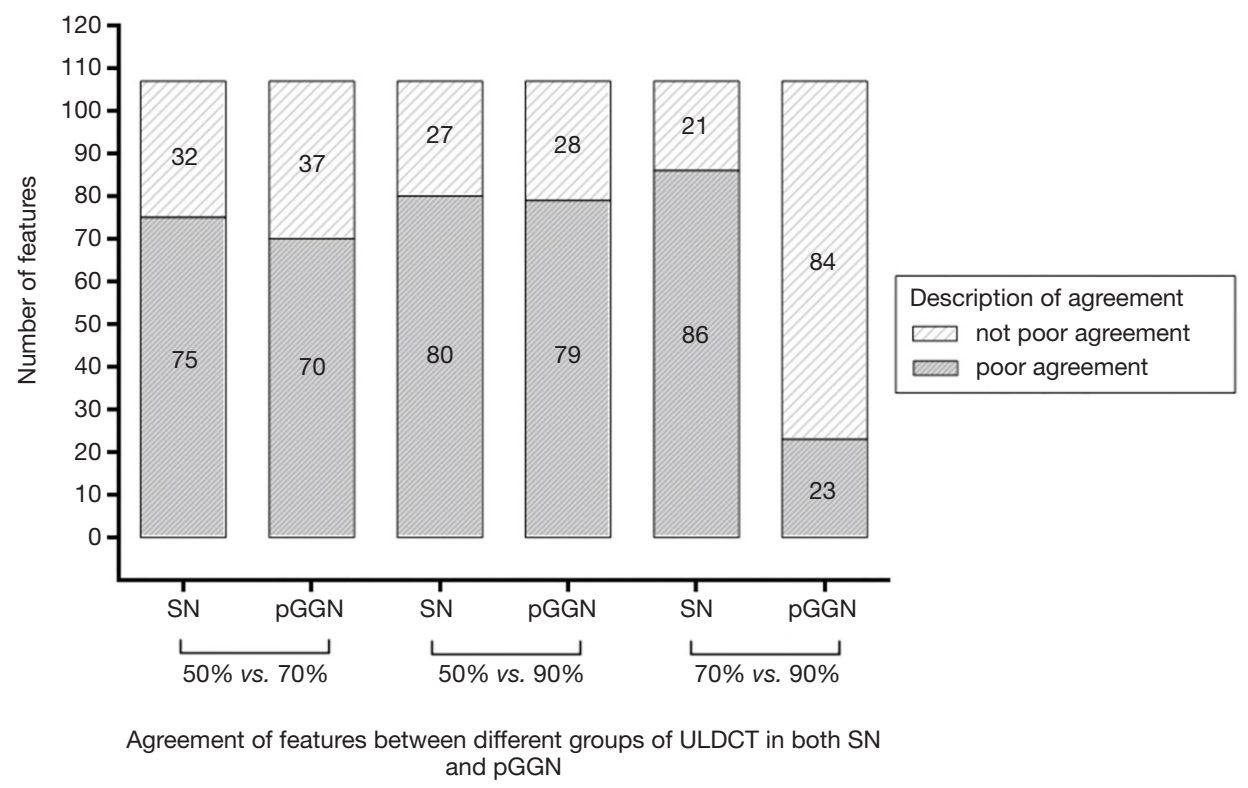

Figure 2 Agreement of all features between different groups of ultra-low-dose CT (ULDCT) reconstructed with different ASiR-V levels in both solid nodule (SN) and pure ground-glass nodule (pGGN).

\section{Statistical analysis}

Statistical analyses were performed using SPSS version 26.0 (IBM) and MedCalc version 19.0.7. $\mathrm{P}<0.05$ (two-sided) was considered significant. All radiomic features were regarded as continuous in nature. Continuous variables are reported as mean \pm standard deviation, and categorical variables as frequencies or percentages. The concordance correlation coefficient (CCC) (30) was calculated to describe the agreement among ULDCTs with different ASiR-V levels and the agreement between ULDCTs with different ASiR-V levels and LDCT for each feature. CCC values of $>0.99,0.95-0.99,0.91-0.95$, and $<0.90$ denoted "almost perfect", "substantial", "moderate", and "poor" agreement, respectively (31). Chi-square statistics were used to compare differences in the proportion of features with CCC $>0.9$ among ULDCTs with different ASiR-V levels and between ULDCTs with different ASiR-V levels and LDCT. A paired sample t-test was used to compare differences in CCC values for all features between ULDCTs with different ASiR-V levels and LDCT. The $95 \%$ confidence intervals of mean CCC values were also calculated.

\section{Results}

A total of 111 nodules were analyzed, including 63 SNs and 48 pGGNs. The mean diameter of SNs was $5.2 \pm 1.5$ (4.0-
10.4) $\mathrm{mm}$, and that of pGGNs was $6.1 \pm 1.8(4.0-12.0) \mathrm{mm}$. The ED of ULDCT $(0.15 \pm 0.024 \mathrm{mSv})$ was $78.6 \%$ lower than that of LDCT $(0.7 \pm 0.16 \mathrm{mSv})$.

\section{Agreement between ULDCTs with different ASiR-V levels for all features}

The agreement between ULDCTs with different ASiR-V levels for all features in both SNs and pGGNs is shown in Figure 2. In SNs, the proportion of features with CCC $>0.9$

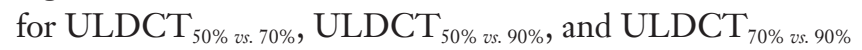
was $29.9 \%, 25.2 \%$, and $19.6 \%$, respectively. There was no obvious difference among them $(\mathrm{P}=0.220)$.

In pGGNs, features with CCC $>0.9$ in ULDCT $_{70 \% \text { vs. } 90 \%}$ accounted for $78.5 \%$ of all features, significantly higher than that of $\mathrm{ULDCT}_{50 \% \text { vs.70\% }}(34.6 \%)$ and $\mathrm{ULDCT}_{50 \% \text { vs. } 90 \%}$ $(26.2 \%)(\mathrm{P}<0.001)$. There was no statistical difference in the proportion between $\mathrm{ULDCT}_{50 \% \text { vs. } 70 \%}$ and $\mathrm{ULDCT}_{50 \% \text { vs. } 90 \%}$ $(\mathrm{P}=0.181)$.

\section{Agreement between ULDCTs with different ASiR-V levels and LDCT}

The agreement between ULDCTs with different ASiR-V levels and LDCT for all features in both SNs and pGGNs is shown in Table 1. In SNs, the number of features with 
Table 1 Agreement between ULDCT and LDCT for all features in both SN and pGGN

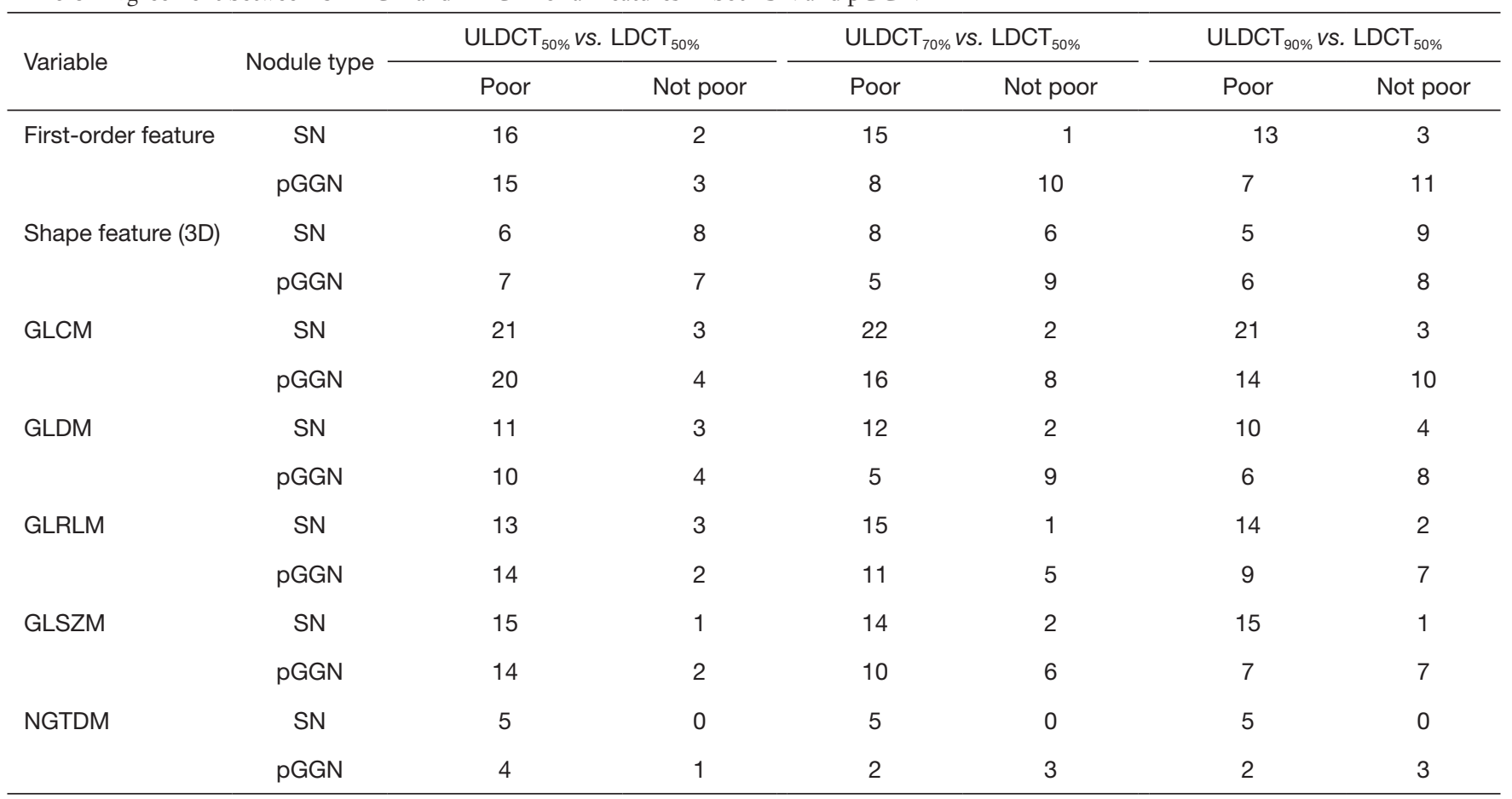

ULDCT, ultra-low-dose computed tomography; LDCT, low-dose computed tomography; SN, solid nodule; pGGN, pure ground-glass nodule; ULDCT ${ }_{50 \%}$, ULDCT with ASiR-V 50\%; ULDCT $70 \%$, ULDCT with ASiR-V 70\%; ULDCT $90 \%$, ULDCT with ASiR-V 90\%; LDCT $50 \%$, LDCT with ASiR-V 50\%; GLCM, gray-level co-occurrence matrix; GLDM, grey-level difference matrix; GLRLM, gray-level run length matrix; GLSZM, grey-level size-zone matrix; NGTDM, neighborhood grey-tone difference matrix; poor represents poor agreement between this group and LDCT; not poor represents not poor agreement between this group and LDCT.

Table 2 Comparison of proportions of features with CCC $>0.9$ between ULDCT with different ASiR-V levels and LDCT

\begin{tabular}{lccccc}
\hline & ULDCT $_{50 \%}$ vs. $\mathrm{LDCT}_{50 \%}$ & ULDCT $_{70 \%}$ vs. $\mathrm{LDCT}_{50 \%}$ & ULDCT $_{90 \%}$ vs. LDCT $_{50 \%}$ & $\chi^{2}$-value & P value \\
\hline SN & $18.7 \%(20 / 107)$ & $13.1 \%(14 / 107)$ & $20.6 \%(22 / 107)$ & 2.250 \\
pGGN & $21.5 \%(23 / 107)^{*}$ & $46.7 \%(50 / 107)$ & $50.5 \%(54 / 107)$ & 22.227 & $<0.001$ \\
\hline
\end{tabular}

ULDCT, ultralow-dose computed tomography; LDCT, low-dose computed tomography; CCC, concordance correlation coefficient; ULDCT $_{50 \%}$, ULDCT with ASiR-V 50\%; ULDCT $70 \%$, ULDCT with ASiR-V 70\%; ULDCT $90 \%$, ULDCT with ASiR-V 90\%; LDCT $50 \%$, LDCT with ASiR-V 50\%; SN, solid nodule; pGGN, pure ground-glass nodule. *The result of this group was different from the other two groups.

different agreements between $\mathrm{ULDCT}_{50 \%}$ and $\mathrm{LDCT}_{50 \%}$ was 87 for poor agreement, and 20 for not poor agreement. Between $\mathrm{ULDCT}_{70 \%}$ and $\mathrm{LDCT}_{50 \%}$, there were 93 features with poor agreement, and 14 with not poor agreement. Between ULDCT $_{90 \%}$ and LDCT $_{50 \%}$, the number of features was 85 for poor agreement, and 22 for not poor agreement.

In pGGNs, the number of features with different agreements between $\mathrm{ULDCT}_{50 \%}$ and $\mathrm{LDCT}_{50 \%}$ was 84 for poor agreement, and 23 for not poor agreement. Between ULDCT $_{70 \%}$ and $\mathrm{LDCT}_{50 \%}$, there were 57 features with poor agreement, and 50 with not poor agreement. Between
$\mathrm{ULDCT}_{90 \%}$ and $\mathrm{LDCT}_{50 \%}$, the number of features was 54 for not poor agreement, and 53 for poor agreement.

\section{Proportion of features with CCC $>0.9$ between ULDCTs with different ASiR-V levels and LDCT}

The comparison of proportions of features with CCC $>0.9$ between ULDCTs with different ASiR-V levels and LDCT is shown in Table 2. In SNs, there was no statistical difference for the proportion of features with CCC $>0.9$ between ULDCTs with different ASiR-V levels and LDCT 
Table 3 Comparison of mean CCC values for the agreement of all features between ULDCT with different ASiR-V levels and LDCT

\begin{tabular}{|c|c|c|c|c|c|}
\hline Variable & Group 1 & Group 2 & Group 3 & $t$-value & $P$ value \\
\hline
\end{tabular}

ULDCT, ultralow-dose computed tomography; LDCT, low-dose computed tomography; SN, solid nodule; pGGN, pure ground-glass nodule; CCC, concordance correlation coefficient; Cl, confidence interval; Group 1, agreement between ULDCT with ASiR-V $50 \%$ vs. LDCT with ASiR-V 50\%; Group 2, agreement between ULDCT with ASiR-V 70\% vs. LDCT with ASiR-V 50\%; Group 3, agreement between ULDCT with ASiR-V 90\% vs. LDCT with ASiR-V 50\%. ${ }^{2}$ Paired samples t-test for Group 1 and Group 2; ${ }^{b}$ Paired samples t-test for Group 1 and Group 3; ' $P$ aired samples t-test for Group 2 and Group 3.

$(\mathrm{P}=0.325)$. In pGGNs, the proportion of features with CCC $>0.9$ between ULDCT $_{70 \%}$ and LDCT $_{50 \%}$ was almost the same as that between $\mathrm{ULDCT}_{90 \%}$ and $\mathrm{LDCT}_{50 \%}(\mathrm{P}=0.584)$. The two proportions above were all higher than that between $\mathrm{ULDCT}_{50 \%}$ and $\mathrm{LDCT}_{50 \%}(\mathrm{P}<0.001)$.

\section{Comparison of mean CCC values between ULDCTs with different ASiR-V levels and LDCT}

The comparison of mean CCC values between ULDCTs with different ASiR-V levels and LDCT in both SNs and pGGNs is shown in Table 3. In SNs, the mean CCC for the agreement between $\mathrm{ULDCT}_{90 \%}$ and $\mathrm{LDCT}_{50 \%}$ was $0.67 \pm 0.26$, which was not statistically different from that for $\mathrm{ULDCT}_{50 \%} v s . \mathrm{LDCT}_{50 \%}(0.68 \pm 0.24)$ and that for $\mathrm{ULDCT}_{70 \%}$ vs. $\mathrm{LDCT}_{50 \%}(0.64 \pm 0.21)(\mathrm{P}>0.05)$. However, the mean CCC between $\mathrm{ULDCT}_{50 \%}$ and $\mathrm{LDCT}_{50 \%}$ was slightly higher than that between $\mathrm{ULDCT}_{70 \%}$ and $\mathrm{LDCT}_{50 \%}$ $(\mathrm{P}<0.001)$.

In pGGNs, the mean CCC for the agreement between $\mathrm{ULDCT}_{90 \%}$ and $\mathrm{LDCT}_{50 \%}$ was $0.79 \pm 0.19$, which was higher than that for $\mathrm{ULDCT}_{70 \%} v s$. $\mathrm{LDCT}_{50 \%}(0.76 \pm 0.24)$ and that for $\mathrm{ULDCT}_{50 \%} v s$. $\mathrm{LDCT}_{50 \%}(0.61 \pm 0.28)(\mathrm{P}<0.05)$. Additionally, the mean CCC between ULDCT $_{70 \%}$ and $\mathrm{LDCT}_{50 \%}$ was also higher than that between $\mathrm{ULDCT}_{50 \%}$ and $\mathrm{LDCT}_{50 \%}(\mathrm{P}<0.001)$.

\section{Discussion}

To our knowledge, this is the first study to show the effect of ASiR-V levels on the reproducibility of CT radiomic features between ULDCT and LDCT. In this study, we found that ASiR-V levels had a pronounced effect on the radiomic features of ULDCT (including first-order statistical, texture, and structural features), with pGGNs being more significantly affected than SNs. Meanwhile, the increase in ASiR-V levels could enhance the reproducibility of radiomic features between ULDCT and LDCT.

Because of the differences in acquisition parameters and reconstruction techniques of CT images, there was controversial about the reproducibility of $\mathrm{CT}$ radiomic features. In a study on the effect of reconstruction algorithms on CT radiomic features of pulmonary tumors, Kim et al. (32) found that 66.7 (4/6) first-order tumor intensity features and $75 \%(3 / 4)$ GLCM features were significantly influenced by the noise reduction strength of Sinogram Affirmed Iterative Reconstruction (SAFIRE) from level 3 to level 5. In the meantime, Prezzi et al. (21) investigated whether ASiR affected CT radiomic quantification in primary colorectal cancer and found that incremental ASiR levels determined a significant change in most statistical radiomic features. Our results also indicated that the ULDCT radiomic features would be strongly influenced by the change in ASiR-V levels in both $\mathrm{SN}$ and pGGN. There were at most 86 features with $\mathrm{CCC}<0.9$ in $\mathrm{SN}$, and the number in pGGN reached at most 79, which was more than $73 \%$ of the total features. These studies indicated that $\mathrm{CT}$ radiomic quantification would be markedly influenced by the change in ASiR levels, similar to other IR algorithms such as SAFIRE and ASiR.

Except for reconstruction algorithms, the reproducibility of CT radiomic features will also be influenced by the radiation dose of CT scan (33-35). In a study on the influence of radiation dose and CT reconstruction setting on the reproducibility of CT radiomic features within the same patient, Meyer et al. (33) found that radiation dose had an obvious effect on the reproducibility of radiomic features, and the percentage of radiomic features deemed reproducible was reduced to $18 \%(19 / 106)$ from the dose level $100 \%$ to dose level $25 \%$. Another study also found that 
the reduction of radiation dose from $120 \mathrm{mAs}$ to $30 \mathrm{mAs}$ led to significant changes in 90\% (18/20) radiomic features (34). In our study, the reproducibility of radiomic features between $\mathrm{ULDCT}_{50 \%}$ and $\mathrm{LDCT}_{50 \%}$ markedly decreased when the ED of ULDCT was reduced to approximately $20 \%$ of LDCT. The mean CCC value for the agreement of all features between ULDCT $\mathrm{U}_{50 \%}$ and $\mathrm{LDCT}_{50 \%}$ was approximately 0.68 in SN and 0.61 in pGGN. In addition, the proportion of features with CCC $<0.9$ was as high as $78.5 \%$ to $81.3 \%$, which aligned with the results of the two studies above. All these results suggest that the reproducibility of radiomic features between ULDCT and LDCT will significantly decrease with a reduction in radiation dose.

It is well known that the noise of an image will increase with the reduction of radiation dose. In our study, the reduction of radiation dose also led to significant changes in the reproducibility of radiomic features between ULDCT and LDCT. However, some studies have indicated that increasing ASiR-V levels can lead to the reduction of image noise and improve the contrast-to-noise ratio (36). In view of this fact, we hypothesized that incremental ASiR-V levels may increase the reproducibility of radiomic features between ULDCT and LDCT.

In our study, we extensively assessed the agreement of radiomic features between ULDCT with different ASiR-V levels and LDCT in both SN and pGGN. In SN, the agreement of radiomic features between ULDCT and LDCT underwent a slight change when ASiR-V levels of ULDCT changed and the radiation dose of the two CT scans remained steady. Despite the ASiR-V level up to $90 \%$ from $50 \%$, the magnitude of variation in the mean CCC value for the agreement of all features between ULDCT and LDCT was less than 0.04 . In addition, the proportion of features with CCC $>0.9$ between ULDCT with different ASiR-V levels and LDCT did not strongly increase.

However, the agreement of radiomic features between ULDCT and LDCT increased greatly with increasing ASiR-V levels for ULDCT in pGGN. The mean CCC value for the agreement of all features between ULDCT $_{90 \%}$ and $\mathrm{LDCT}_{50 \%}$ increased by 0.18 compared with that between $\mathrm{ULDCT}_{50 \%}$ and $\mathrm{LDCT}_{50 \%}$, with a $30 \%$ increase. Although the ED of ULDCT was only $21.4 \%$ that of LDCT, the mean CCC value for the agreement of all features between $\mathrm{ULDCT}_{90 \%}$ and $\mathrm{LDCT}_{50 \%}$ was as high as 0.79. In the meantime, the proportion of features with CCC $>0.9$ between ULDCT $_{90 \%}$ and LDCT $_{50 \%}$ was up to $50.5 \%$ compared with that between $\mathrm{ULDCT}_{50 \%}$ and $\mathrm{LDCT}_{50 \%}$.
For the obviously different effect between SN and pGGN, it may be explained that the lower intensity of pGGN was more sensitive to changes in image noise. In conclusion, the results of our study indicate that incremental ASiR-V levels, to some degree, can reduce the negative effect of dose reduction on the reproducibility of LDCT radiomic features and enhance the reproducibility of radiomic features between ULDCT and LDCT. Meanwhile, it has been recently shown that CT image reconstruction algorithms not only affect the reproducibility of radiomic features, but also affect the diagnostic performance of radiomics models (37). Thus, the difference in diagnostic performance of the radiomics models between LDCT and ULDCT may be narrowed to some extent by increasing ASiR-V levels to enhance the reproducibility of radiomic features.

This study had some limitations. First, levels of ASiR-V for ULDCT in our study only included $50 \%, 70 \%$, and $90 \%$, which did not cover the range from $0 \%$ to $100 \%$. Afadzi et al. (16) recommended that ASiR-V levels below $70 \%$ may be appropriate for LDCT and ULDCT. Besides, levels of ASiR-V from $40 \%$ to $60 \%$ were recommended as the reconstruction levels for chest CT in another study (36). Thus, we chose the most likely levels of ASiR-V (including $50 \%, 70 \%$, and $90 \%$ ), which may be used for LDCT and ULDCT in practical applications. Second, our study only focused on the comparison of reproducibility of radiomic features between ULDCT and LDCT in both SNs and pGGNs, not covering PSNs. Third, whether the difference in reproducibility of radiomic features between ULDCT and LDCT can affect the diagnostic performance of radiomics models based on images of two scans needs to be explored. Therefore, more types of pulmonary nodules need to be included and the effect of ASiR-V levels on the diagnostic performance of radiomics models based on images of ULDCT and LDCT should be investigated in the future.

\section{Conclusions}

In conclusion, we have confirmed that ASiR-V levels can also significantly affect the quantification of radiomics features computed at ULDCT in pulmonary nodules, similar to the radiation dose, and that pGGN was more sensitive than SN. The increase in ASiR-V levels could enhance the reproducibility of radiomic features between ULDCT and LDCT to some degree. In the future, higher ASiR-V levels may be taken when radiomics models 
based on the ULDCT images are used in the diagnosis of pulmonary nodules.

\section{Acknowledgments}

We would like to thank Editage (www.editage.cn) for English language editing.

Funding: None.

\section{Footnote}

Conflicts of Interest: All authors have completed the ICMJE uniform disclosure form (available at http://dx.doi. org/10.21037/qims-20-932). The authors have no conflicts of interest to declare

Ethical Statement: This study was approved by the institutional review board of our department (NO. M2019296), and informed consent was obtained from the participants.

Open Access Statement: This is an Open Access article distributed in accordance with the Creative Commons Attribution-NonCommercial-NoDerivs 4.0 International License (CC BY-NC-ND 4.0), which permits the noncommercial replication and distribution of the article with the strict proviso that no changes or edits are made and the original work is properly cited (including links to both the formal publication through the relevant DOI and the license). See: https://creativecommons.org/licenses/by-nc-nd/4.0/.

\section{References}

1. Gillies RJ, Kinahan PE, Hricak H. Radiomics: Images Are More than Pictures, They Are Data. Radiology 2016;278:563-77.

2. Kim H, Park CM, Goo JM, Wildberger JE, Kauczor HU. Quantitative Computed Tomography Imaging Biomarkers in the Diagnosis and Management of Lung Cancer. Invest Radiol 2015;50:571-83.

3. Fan L, Fang M, Li Z, Tu W, Wang S, Chen W, Tian J, Dong D, Liu S. Radiomics signature: a biomarker for the preoperative discrimination of lung invasive adenocarcinoma manifesting as a ground-glass nodule. Eur Radiol 2019;29:889-97.

4. Koyasu S, Nishio M, Isoda H, Nakamoto Y, Togashi $\mathrm{K}$. Usefulness of gradient tree boosting for predicting histological subtype and EGFR mutation status of non- small cell lung cancer on 18F FDG-PET/CT. Ann Nucl Med 2020;34:49-57.

5. Beig N, Khorrami M, Alilou M, Prasanna P, Braman N, Orooji M, Rakshit S, Bera K, Rajiah P, Ginsberg J, Donatelli C, Thawani R, Yang M, Jacono F, Tiwari P, Velcheti V, Gilkeson R, Linden P, Madabhushi A. Perinodular and Intranodular Radiomic Features on Lung CT Images Distinguish Adenocarcinomas from Granulomas. Radiology 2019;290:783-92.

6. Wilson R, Devaraj A. Radiomics of pulmonary nodules and lung cancer. Transl Lung Cancer Res 2017;6:86-91.

7. Thawani R, McLane M, Beig N, Ghose S, Prasanna P, Velcheti V, Madabhushi A. Radiomics and radiogenomics in lung cancer: A review for the clinician. Lung Cancer 2018;115:34-41.

8. Aberle DR, Adams AM, Berg CD, Black WC, Clapp JD, Fagerstrom RM, Gareen IF, Gatsonis C, Marcus PM, Sicks JD. Reduced lung-cancer mortality with lowdose computed tomographic screening. $\mathrm{N}$ Engl J Med 2011;365:395-409.

9. Delzell DA, Magnuson S, Peter T, Smith M, Smith BJ. Machine Learning and Feature Selection Methods for Disease Classification With Application to Lung Cancer Screening Image Data. Front Oncol 2019;9:1393.

10. Mao L, Chen H, Liang M, Li K, Gao J, Qin P, Ding X, Li X, Liu X. Quantitative radiomic model for predicting malignancy of small solid pulmonary nodules detected by low-dose CT screening. Quant Imaging Med Surg 2019;9:263-72.

11. Hawkins S, Wang H, Liu Y, Garcia A, Stringfield O, Krewer H, Li Q, Cherezov D, Gatenby RA, Balagurunathan Y, Goldgof D, Schabath MB, Hall L, Gillies RJ. Predicting Malignant Nodules from Screening CT Scans. J Thorac Oncol 2016;11:2120-8.

12. Ohno Y, Koyama H, Seki S, Kishida Y, Yoshikawa T. Radiation dose reduction techniques for chest CT: Principles and clinical results. Eur J Radiol 2019;111:93-103.

13. Lell MM, Kachelrieß M. Recent and Upcoming Technological Developments in Computed Tomography: High Speed, Low Dose, Deep Learning, Multienergy. Invest Radiol 2020;55:8-19.

14. Nishio M, Koyama H, Ohno Y, Negi N, Seki S, Yoshikawa T, Sugimura K. Emphysema Quantification Using Ultralow-Dose CT With Iterative Reconstruction and Filtered Back Projection. AJR Am J Roentgenol 2016;206:1184-92.

15. Euler A, Solomon J, Marin D, Nelson RC, Samei E. A Third-Generation Adaptive Statistical Iterative 
Reconstruction Technique: Phantom Study of Image Noise, Spatial Resolution, Lesion Detectability, and Dose Reduction Potential. AJR Am J Roentgenol 2018;210:1301-8.

16. Afadzi M, Lysvik EK, Andersen HK, ACT M. Ultralow dose chest computed tomography: Effect of iterative reconstruction levels on image quality. Eur J Radiol 2019;114:62-8.

17. Messerli M, Kluckert T, Knitel M, Wälti S, Desbiolles L, Rengier F, Warschkow R, Bauer RW, Alkadhi H, Leschka $\mathrm{S}$, Wildermuth S. Ultralow dose CT for pulmonary nodule detection with chest $\mathrm{x}$-ray equivalent dose - a prospective intra-individual comparative study. Eur Radiol 2017;27:3290-9.

18. Jin S, Zhang B, Zhang L, Li S, Li S, Li P. Lung nodules assessment in ultra-low-dose CT with iterative reconstruction compared to conventional dose CT. Quant Imaging Med Surg 2018;8:480-90.

19. Ye K, Zhu Q, Li M, Lu Y, Yuan H. A feasibility study of pulmonary nodule detection by ultralow-dose CT with adaptive statistical iterative reconstruction- $\mathrm{V}$ technique. Eur J Radiol 2019;119:108652.

20. Miller AR, Jackson D, Hui C, Deshpande S, Kuo E, Hamilton GS, Lau KK. Lung nodules are reliably detectable on ultra-low-dose CT utilising model-based iterative reconstruction with radiation equivalent to plain radiography. Clin Radiol 2019;74:409.e17-409.e22.

21. Prezzi D, Owczarczyk K, Bassett P, Siddique M, Breen DJ, Cook GJR, Goh V. Adaptive statistical iterative reconstruction (ASIR) affects CT radiomics quantification in primary colorectal cancer. Eur Radiol 2019;29:5227-35.

22. Kim H, Goo JM, Ohno Y, Kauczor HU, Hoffman EA, Gee JC, Beek EJRV. Effect of Reconstruction Parameters on the Quantitative Analysis of Chest Computed Tomography. J Thorac Imaging 2019;34:92-102.

23. Zhao W, Zhang W, Sun Y, Ye Y, Yang J, Chen W, Gao P, Li J, Li C, Jin L, Wang P, Hua Y, Li M. Convolution kernel and iterative reconstruction affect the diagnostic performance of radiomics and deep learning in lung adenocarcinoma pathological subtypes. Thorac Cancer 2019;10:1893-903.

24. Solomon J, Mileto A, Nelson RC, Roy CK, Samei E. Quantitative Features of Liver Lesions, Lung Nodules, and Renal Stones at Multi-Detector Row CT Examinations: Dependency on Radiation Dose and Reconstruction Algorithm. Radiology 2016;279:185-94.

25. Midya A, Chakraborty J, Gönen M, RKG D, Simpson AL. Influence of CT acquisition and reconstruction parameters on radiomic feature reproducibility. J Med Imaging (Bellingham) 2018;5:011020.

26. Mileto A, Guimaraes LS, McCollough CH, Fletcher JG, Yu L. State of the Art in Abdominal CT: The Limits of Iterative Reconstruction Algorithms. Radiology 2019;293:491-503.

27. MacMahon H, Naidich DP, Goo JM, Lee KS, Leung ANC, Mayo JR, Mehta AC, Ohno Y, Powell CA, Prokop M, Rubin GD, Schaefer-Prokop CM, Travis WD, Schil PEV, Bankier AA. Guidelines for Management of Incidental Pulmonary Nodules Detected on CT Images: From the Fleischner Society 2017. Radiology 2017;284:228-43.

28. Deak PD, Smal Y, Kalender WA. Multisection CT protocols: sex- and age-specific conversion factors used to determine effective dose from dose-length product. Radiology 2010;257:158-66.

29. Lambin P, Rios-Velazquez E, Leijenaar R, Carvalho S, Stiphout RGPMV, Granton P, Zegers CML, Gillies R, Boellard R, Dekker A, Aerts HJWL. Radiomics: extracting more information from medical images using advanced feature analysis. Eur J Cancer 2012;48:441-6.

30. Liu J, Tang W, Chen G, Lu Y, Feng C, Tu XM. Correlation and agreement: overview and clarification of competing concepts and measures. Shanghai Arch Psychiatry 2016;28:115-20.

31. McBride GB (2005). A proposal for strength-of-agreement criteria for Lin's concordance correlation coefficient. NIWA Client Report: HAM2005-062. Available online: http://www.medcalc.org/download/pdf/McBride2005.pdf. Accessed 18 Dec 2020.

32. Kim H, Park CM, Lee M, Park SJ, Song YS, Lee JH, Hwang EJ, Goo JM. Impact of Reconstruction Algorithms on CT Radiomic Features of Pulmonary Tumors: Analysis of Intra- and Inter-Reader Variability and Inter-Reconstruction Algorithm Variability. PLoS One 2016;11:e0164924.

33. Meyer M, Ronald J, Vernuccio F, Nelson RC, RamirezGiraldo JC, Solomon J, Patel BN, Samei E, Marin D. Reproducibility of CT Radiomic Features within the Same Patient: Influence of Radiation Dose and CT Reconstruction Settings. Radiology 2019;293:583-91.

34. Kim YJ, Lee HJ, Kim KG, Lee SH. The Effect of CT Scan Parameters on the Measurement of CT Radiomic Features: A Lung Nodule Phantom Study. Comput Math Methods Med 2019;2019:8790694.

35. Buch K, Li B, Qureshi MM, Kuno H, Anderson SW, Sakai O. Quantitative Assessment of Variation in CT Parameters 
on Texture Features: Pilot Study Using a Nonanatomic Phantom. AJNR Am J Neuroradiol 2017;38:981-5.

36. Tang H, Yu N, Jia Y, Yu Y, Duan H, Han D, Ma G, Ren $\mathrm{C}, \mathrm{He}$ T. Assessment of noise reduction potential and image quality improvement of a new generation adaptive statistical iterative reconstruction (ASIR-V) in chest CT.

Cite this article as: Ye K, Chen M, Zhu Q, Lu Y, Yuan H. Effect of adaptive statistical iterative reconstruction-V (ASiR-V) levels on ultra-low-dose CT radiomics quantification in pulmonary nodules. Quant Imaging Med Surg 2021;11(6):23442353. doi: 10.21037 /qims-20-932
Br J Radiol 2018;91:20170521.

37. Kim H, Park CM, Gwak J, Hwang EJ, Lee SY, Jung J, Hong H, Goo JM. Effect of CT Reconstruction Algorithm on the Diagnostic Performance of Radiomics Models: A Task-Based Approach for Pulmonary Subsolid Nodules. AJR Am J Roentgenol 2019;212:505-12. 
Supplementary

Table S1 Summary and brief description of all the 107 CT radiomic features extracted

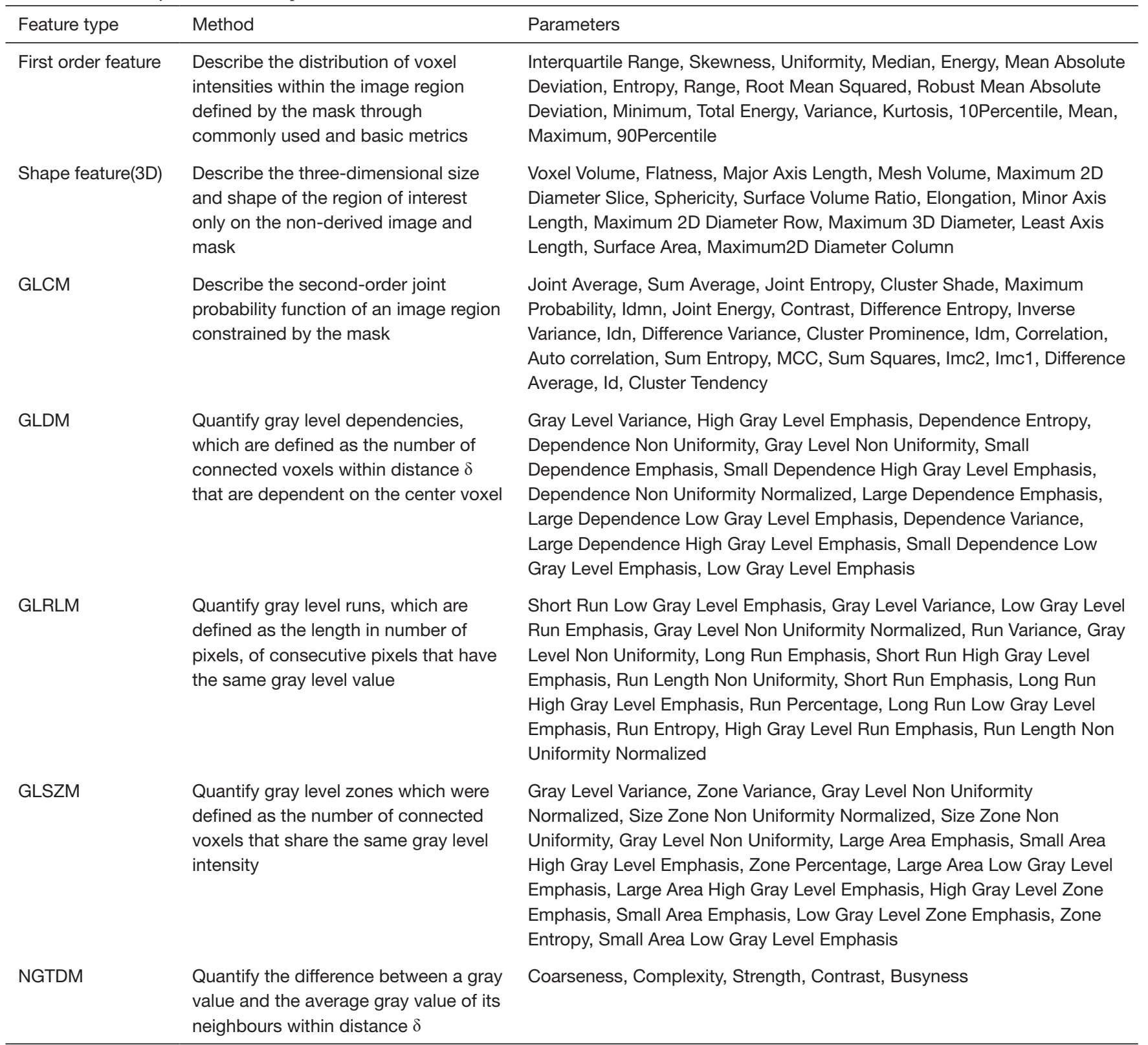

GLCM, gray level co-occurrence matrix; GLDM, gray level dependence matrix; GLRLM, gray level run length matrix; GLSZM, gray level size zone matrix; NGTDM, neighbouring gray tone difference matrix. 